\title{
BOLSAS DE VALORES: RENTABILIDAD Y RIESGO
}

\author{
STOCK EXCHANGES: RENTABILITY AND RISK
}

\author{
${ }^{1}$ Elias Felix Huertas Camones
}

\begin{abstract}
Resumen
Las bolsas de valores del mundo constituyen una de las alternativas atractiva de inversión en el mundo. En el caso de Perú, debido a las escasas alternativas de inversión que tenemos, es necesario considerar la posibilidad de invertir en otros países. Para evaluar las alternativas de inversión, tanto locales como del exterior, es necesario conocer por lo menos la rentabilidad y el riesgo de cada alternativa. Por esta razón esta investigación tuvo como propósito principal determinar la relación existente entre riesgo y rentabilidad en los índice generales de las Bolsas de Valores del mundo en la última década, se calculó el riesgo y rentabilidad de cada una de las bolsas del mundo, y finalmente se trató de determinar las diferencias entre las regiones del mundo. La base de datos para esta investigación estuvo constituida por la serie histórica mensual de los índices generales de bolsa de los diferentes países. Estos datos fueron procesados utilizando el software PH stat2. Las principales conclusiones a las que se llegó fueron que existe una relación lineal positiva entre riesgo y rentabilidad de las Bolsas de Valores del Mundo, las Bolsas De Valores de la región Asia Pacífico fueron las más rentables y las de mayor riesgo, las de Américas presentaron riesgo y rentabilidad intermedia y las de Europa y África Medio Este mostraron los menor niveles de riesgo y de rentabilidad.
\end{abstract}

Palabras clave: Bolsa de valores, índice general, rentabilidad y riesgo.

\begin{abstract}
The stock markets of the world are one of the attractive investment alternatives in the world. In the case of Peru, due to limited investment alternatives that we have, we must consider the possibility of investing in other countries. To evaluate investment alternatives, both local and from abroad, we need to know at least the return and risk of each alternative. Therefore this research had as main purpose to determine the relationship between risk and return in the general index of the Stock Exchanges of the world in the last decade, the risk and return of each of the world's stock was calculated, and finally it sought to determine the differences between the regions. The database for this study consisted of monthly time series of the broad stock market indices of different countries. These data were processed using the software PH STAT2. The main conclusions reached were that there is a positive linear relationship between risk and return of the stock exchanges of the world, the Stock Exchanges of the Asia Pacific region were the most profitable and riskier, the Americas presented intermediate risk and profitability and Africa Europe and the Middle East showed the lowest levels of risk and return.
\end{abstract}

Key words: Stock exchange, broad index, return and risk.

\section{Introducción}

Las bolsas de valores del mundo constituyen una de las alternativas atractiva de inversión del mundo. En el caso de nuestro país Perú, debido a las escasas alternativas de inversión que tenemos, es necesario considerar la posibilidad de invertir en otros países. Para evaluar las alternativas de inversión, tanto locales como del exterior, es necesario conocer por lo menos la rentabilidad y el riesgo de cada alternativa.

Como toda alternativa de inversión, la inversión en las bolsas de valores está sujeta al riesgo; por lo tanto, si se mide la rentabilidad de una inversión también se debe medir el riesgo asociado que conlleva. Desde el punto de vista financiero se afirma que para obtener mayor rentabilidad hay que estar dispuesto a aceptar un mayor nivel del riesgo. Basado en esta afirmación, en esta investigación se trata de indagar si lo mencionado también se cumple para las inversiones en las Bolsas de Valores.

El Problema general que se abordara en esta investigación es ¿Qué relación existe entre riesgo y rentabilidad en las bolsas de valores?, los problemas específicos a abordar son: ¿Cuál es la rentabilidad de las Bolsas de Valores?, ¿Cuál es el riesgo de las Bolsas de Valores? Y si ¿Existe diferencias entre la rentabilidad y riesgo de las Bolsas de Valores de las diferentes regiones del mundo?

Por lo tanto, el objetivo general de esta investigación es determinar la relación que existe entre riesgo y 
rentabilidad de las Bolsas de Valores. Los objetivos específicos son: determinar la rentabilidad de las Bolsas de Valores, determinar el riesgo de las Bolsas de Valores y determinar las diferencias entre la rentabilidad y riesgo de las Bolsas de Valores de las diferentes regiones del mundo.

\section{Materiales y método}

La presente investigación se llevó a cabo en la Universidad Nacional Agraria La Molina, en el distrito de la Molina, en la provincia y región de Lima, Perú.

La hipótesis general de investigación fue: la rentabilidad y riesgo de las Bolsas de Valores presentan una asociación positiva.

La presente investigación es descriptiva porque describe la situación de la rentabilidad y riesgo de las diversas Bolsas de Valores del mundo. Es correlacional por que trata de determinar el grado de asociación entre la variables riesgo y rentabilidad de las Bolsas de Valores del Mundo.

La fuente de datos de los índices generales de Bolsas fueron los reportes mensuales de los índices generales de cada una de las Bolsas De Valores del Mundo. Estos datos están basados en los reportes mensuales de las Bolsas de Valores de cada país así como de las estadísticas de la Federación Mundial de Mercados de Valores WFE². El periodo de tiempo considerado comprende la última década, es decir desde el año 2006 hasta Junio del año 2015.

La metodología consistió en primer lugar en obtener la base de datos de índices generales de las Bolsas de fuentes secundarias, luego se procedió a calcular el riesgo y rentabilidad de cada Bolsa o mejor dicho de cada índice general de cada Bolsa. Enseguida, se procedió a realizar la comparación del promedio de rentabilidad y riesgo entre los índices general de la Bolsas de las diferentes regiones del mundo. Finalmente, se procedió a correlacionar el riesgo y la rentabilidad de las bolsas del mundo. Es necesario aclarar que las rentabilidades promedio anual se obtuvieron mediante la capitalización compuesta de las rentabilidades promedio.

Basado en Ross et $\mathrm{al}^{3}$ (2012) y Brealey et $\mathrm{al}^{4}$ (2010), los cálculos de riesgo y rentabilidad se realizaron con las siguientes modalidades y formulas:

a) Rentabilidad mensual, $\mathrm{R}=[\{\mathrm{Pi}-\mathrm{P}(\mathrm{i}-1)\} / \mathrm{P}(\mathrm{i}-1)]^{*} 100$

Dónde:

$\mathrm{R}=$ Rentabilidad.

$\mathrm{Pi}=$ Precio en el periodo.

$\mathrm{P}(\mathrm{i}-1)=$ Precio del periodo anterior.

\footnotetext{
${ }^{2}$ World Federation of Exchanges. http://www.world-exchanges.org/ ${ }^{3}$ Ross, Westerfield y Jaffe. 2012. Finanzas Corporativas. McGraw Hill. ${ }^{4}$ Brealey \& Myers, S. 2011. Fundamentos de Finanzas Corporativas. Mc Graw Hill.
}

b) Rentabilidad promedio, $\mathrm{Rp}=\sum \mathrm{Ri} / \mathrm{n}$

Dónde:

$\mathrm{Ri}=$ Rentabilidad de cada periodo.

$\mathrm{n}=$ número de periodos de la serie.

c) Rentabilidad acumulada del periodo (Ra).

Dónde:

$\mathrm{Ra}=[(\mathrm{Pn}-\mathrm{Po}) / \mathrm{Po}]^{*} 100$.

$\mathrm{Pn}=$ Precio en el periodo final de la serie.

$\mathrm{Po}=$ Precio en el periodo inicial de la serie.

El riesgo se calculó mediante las formulas, ya conocidas, de desviación estándar $(\sigma)$ y Coeficiente de variabilidad (CV).

Para el procesamiento de datos se utilizó Excel y el software PH Stat $2^{5}$.

Para la comparación de los promedios de rentabilidad de las Bolsas entre regiones del mundo, se determinó la rentabilidad y su riesgo por cada región y se procedió a realizar las comparaciones. Las variables utilizadas fueron: rentabilidad promedio, rentabilidad acumulada, riesgo según desviación estándar y también el coeficiente de variabilidad.

Para medir el grado de asociación entre riesgo y rentabilidad se realizó una regresión lineal simple. La variable dependiente fue la rentabilidad y la variable independiente fue el riesgo.

Las Bolsas de Valores y sus respectivos índices generales con los cuales se realizó esta investigación fueron 49 . Además, estos fueron agrupados en tres regiones, de acuerdo a lo considerado por la WFE citada anteriormente, Asia Pacifico, Américas y Europa y África medio este. En el Cuadro 1 se presentan los índices con sus respectivos países y su región.

Es necesario hacer una breve revisión del concepto de índices bursátiles. Según Martin, (2007) “ "un índice bursátil es un parámetro que tiene por objeto medir la evolución de un determinado mercado". Con tal propósito se debe elegir un conjunto de acciones correspondientes a empresas representativas. Un índice bursátil es un indicador que representa la evolución de un conjunto de acciones representativas de esta Bolsa. Lo más importante en un índice es la variación porcentual, es decir si por ejemplo el índice subió 5\% significa que el precio del conjunto de acciones consideradas subió en promedio esa cantidad. A esto índices se les denomina el barómetro de لas Bolsas. Los índices pueden ser generales o específicos. ${ }^{5}$ http://wps.aw.com/phstat/

${ }^{6}$ Martin, Miguel. Mercado de capitales. Thomson 20107. 
Los incides son específicos porque consideran un número limitado de acciones que corresponden a las más representativas, tal como ocurre con el índice selectivos o más conocido como blue chip de la Bolsa de Valores de Lima Perú. Los índices también pueden ser generales porque comprenden una cantidad mayor de acciones que corresponde a las acciones más negociadas en el mercado. En el caso de la Bolsa de Valores de Lima el índice selectivo comprende 15 acciones o empresas y el general 38 acciones.

En cuanto a las limitaciones del estudio debemos mencionar que en esta investigación solo se considerado los índices generales. También, es preciso mencionar que el análisis se ha limitado a la última década. Se han considerado 49 índices de Bolsas, algunos como el caso de la Bolsa de Colombia y otros no se pudieron considerar por no contar con datos completos para el periodo considerado y tampoco otros como el índice American SE que dejaron de ser un índice general.

\section{Resultados y discusión}

A continuación se presentan los principales resultados correspondientes a la rentabilidad, y riesgo de los índices de las bolsas de valores de los diferentes países.

\subsection{Rentabilidad}

Según los resultados obtenidos, el índice que presenta la mayor rentabilidad acumulada es FBM Emas Index correspondiente a la Bolsa de Malasia, con una rentabilidad de 1,187.9\%. Esta cifra es significativamente mayor que la rentabilidad del índice que ocupo el segundo lugar con $702 \%$ que pertenece al índice SZSE composite Index de la Bolsa Shenzhen de China. Esto dos índices corresponden a la región Asia Pacifico. El tercer lugar fue ocupado por el índice Composite de la Bolsa de Valores de Buenos Aires, Argentina correspondiente a la región de las Américas.

Las menos rentables fueron el índice General Price que corresponde a la Bolsa Valores de Atenas con una rentabilidad acumulada negativa, es decir una pérdida de $80 \%$, el índice BSX Index De la Bolsa de Valores de Bermuda con $-70 \%$. La tercera menos rentable fue el índice SE Price Index de la Bolsa Wiener Börse de Viena Austria.

Los índices que presentaron una rentabilidad intermedia fueron el índice Kospi de la Bolsa de Valores de Corea con $55 \%$, el índice SMI de la Bolsa de Suiza con $48 \%$, el índice Cdax de la Bolsa Deutsche Börse de Alemania con $44.6 \%$ y el índice Taiex de la Bolsa de Valores de Taiwan que obtuvo $42.7 \%$ de rentabilidad acumulad

Las rentabilidades acumuladas correspondientes a cada uno de los índices de Bolsa se muestran en detalle en el Cuadro 1 y Figura 1
Cuadro 1. Rentabilidad Acumulada 2006 -Junio 2015.

\begin{tabular}{|c|c|c|c|}
\hline Bolsa de Valores & Índice & Región & $\mathrm{Ra}$ \\
\hline Athens Exchange & General Price & EAME & $-80.0 \%$ \\
\hline Bermuda SE & BSX Index & Américas & $-70.0 \%$ \\
\hline Wiener Börse & SE Price Index & EAME & $-34.9 \%$ \\
\hline Luxembourg SE & $\begin{array}{c}\text { Lux General } \\
\text { Price }\end{array}$ & EAME & $-32.9 \%$ \\
\hline Borsa Italiana & $\begin{array}{l}\text { FTSE Italia } \\
\text { MIB Storico }\end{array}$ & EAME & $-29.7 \%$ \\
\hline Malta SE & $\begin{array}{l}\text { MSE Share } \\
\text { Index }\end{array}$ & EAME & $-24.5 \%$ \\
\hline Irish SE & ISEQ Overall & EAME & $-18.5 \%$ \\
\hline Budapest SE & BUMIX & EAME & $-14.2 \%$ \\
\hline $\begin{array}{l}\text { BME Spanish } \\
\text { Exchanges Bilbao }\end{array}$ & $\begin{array}{l}\text { Índice Bolsa } \\
\text { Bilbao } 1999\end{array}$ & EAME & $-11.4 \%$ \\
\hline $\begin{array}{l}\text { BME Spanish } \\
\text { Exchanges Madrid }\end{array}$ & IGBM Index & EAME & $-8.9 \%$ \\
\hline $\begin{array}{l}\text { Japan Exchange } \\
\text { Group - Tokyo }\end{array}$ & TOPIX & $\begin{array}{c}\text { Asia } \\
\text { Pacific }\end{array}$ & $-4.7 \%$ \\
\hline Euronext Lisbon & BVL General & EAME & $-4.4 \%$ \\
\hline $\begin{array}{l}\text { NASDAQ OMX } \\
\text { Nordic Helsinki }\end{array}$ & OMXH & EAME & $-2.0 \%$ \\
\hline $\begin{array}{l}\text { BME Spanish } \\
\text { Exchanges } \\
\text { Barcelona }\end{array}$ & $\begin{array}{l}\text { BCN Global - } \\
100 \text { Index }\end{array}$ & EAME & $0.0 \%$ \\
\hline Egyptian Exchange & EGX 30 Index & EAME & $5.6 \%$ \\
\hline Euronext Paris & SBF 250 & EAME & $6.3 \%$ \\
\hline $\begin{array}{l}\text { Euronext } \\
\text { Amsterdam }\end{array}$ & AAX & EAME & $7.5 \%$ \\
\hline Euronext Brussels & General Price & EAME & $10.6 \%$ \\
\hline Australian SE & $\begin{array}{l}\text { All Ordinary } \\
\text { Price }\end{array}$ & $\begin{array}{l}\text { Asia } \\
\text { Pacific }\end{array}$ & $11.7 \%$ \\
\hline TMX Group & $\begin{array}{l}\text { S\&P/TSX } \\
\text { Composite }\end{array}$ & Américas & $21.9 \%$ \\
\hline Oslo Bers & OSEBXPR & EAME & $22.8 \%$ \\
\hline NYSE & Composite & Américas & $33.3 \%$ \\
\hline BM\&FBOVESPA & Ibovespa & Américas & $38.3 \%$ \\
\hline $\begin{array}{l}\text { BME Spanish } \\
\text { Exchanges Valencia }\end{array}$ & IGBV Index & EAME & $41.9 \%$ \\
\hline Taiwan SE Corp. & TAIEX & $\begin{array}{c}\text { Asia } \\
\text { Pacific }\end{array}$ & $42.7 \%$ \\
\hline Deutsche Börse & CDAX Price & EAME & $44.6 \%$ \\
\hline $\begin{array}{l}\text { SIX Swiss } \\
\text { Exchange }\end{array}$ & SMI & EAME & $48.1 \%$ \\
\hline Korea Exchange & KOSPI & $\begin{array}{c}\text { Asia } \\
\text { Pacific }\end{array}$ & $48.2 \%$ \\
\hline Tel Aviv SE & General & EAME & $55.2 \%$ \\
\hline $\begin{array}{l}\text { NASDAQ OMX } \\
\text { Nordic Stockholm }\end{array}$ & OMXS & EAME & $64.3 \%$ \\
\hline $\begin{array}{l}\text { Hong Kong } \\
\text { Exchanges }\end{array}$ & $\begin{array}{l}\text { S\&P/HKEX } \\
\text { LargeCap } \\
\text { Index }\end{array}$ & $\begin{array}{c}\text { Asia } \\
\text { Pacific }\end{array}$ & $75.2 \%$ \\
\hline $\begin{array}{l}\text { Borsa Istanbul } \\
\text { The Stock }\end{array}$ & ISE 100 Index & EAME & $84.5 \%$ \\
\hline $\begin{array}{l}\text { Exchange of } \\
\text { Thailand }\end{array}$ & SET Index & $\begin{array}{l}\text { Asia } \\
\text { Pacific }\end{array}$ & $97.3 \%$ \\
\hline Santiago SE & IGPA & Américas & $99.5 \%$ \\
\hline $\begin{array}{l}\text { NASDAQ OMX } \\
\text { Nordic Copenhagen }\end{array}$ & OMXC & EAME & $100.0 \%$ \\
\hline NASDAQ OMX & Composite & Américas & $116.3 \%$ \\
\hline
\end{tabular}


Continuación del cuadro 1

\begin{tabular}{|c|c|c|c|}
\hline Lima SE & $\begin{array}{l}\text { Indice General } \\
\text { BVL (IGBVL) }\end{array}$ & Américas & $132.8 \%$ \\
\hline Mexican Exchange & IPC CompMx & Américas & $156.2 \%$ \\
\hline Johannesburg SE & $\begin{array}{c}\text { FTSE/JSE All } \\
\text { Share }\end{array}$ & EAME & $162.4 \%$ \\
\hline $\begin{array}{l}\text { National Stock } \\
\text { Exchange India }\end{array}$ & S\&P CNX 500 & $\begin{array}{l}\text { Asia } \\
\text { Pacific }\end{array}$ & $166.7 \%$ \\
\hline BSE India & S\&P BSE 500 & $\begin{array}{c}\text { Asia } \\
\text { Pacific }\end{array}$ & $172.3 \%$ \\
\hline Colombo SE & CSE All Share & $\begin{array}{c}\text { Asia } \\
\text { Pacific }\end{array}$ & $228.2 \%$ \\
\hline Shanghai SE & $\begin{array}{c}\text { SSE } \\
\text { Composite } \\
\text { Index }\end{array}$ & $\begin{array}{l}\text { Asia } \\
\text { Pacific }\end{array}$ & $240.0 \%$ \\
\hline Philippine SE & $\begin{array}{l}\text { PSE Index } \\
\text { (PSEi) } \\
\text { JSX }\end{array}$ & $\begin{array}{c}\text { Asia } \\
\text { Pacific }\end{array}$ & $252.6 \%$ \\
\hline Indonesia SE & $\begin{array}{l}\text { JSX } \\
\text { Composite } \\
\text { Index }\end{array}$ & $\begin{array}{c}\text { Asia } \\
\text { Pacific }\end{array}$ & $298.5 \%$ \\
\hline $\begin{array}{l}\text { Singapore } \\
\text { Exchange }\end{array}$ & $\begin{array}{l}\text { Straits Times } \\
\text { Index }\end{array}$ & $\begin{array}{l}\text { Asia } \\
\text { Pacific }\end{array}$ & $433.8 \%$ \\
\hline Buenos Aires SE & Composite & Américas & $508.7 \%$ \\
\hline Shenzhen SE & $\begin{array}{c}\text { SZSE } \\
\text { Composite } \\
\text { Index }\end{array}$ & $\begin{array}{l}\text { Asia } \\
\text { Pacific }\end{array}$ & $702.4 \%$ \\
\hline Bursa Malaysia & $\begin{array}{l}\text { FBM Emas } \\
\text { Index }\end{array}$ & $\begin{array}{l}\text { Asia } \\
\text { Pacific }\end{array}$ & $1187.9 \%$ \\
\hline
\end{tabular}

Elaboración propia.

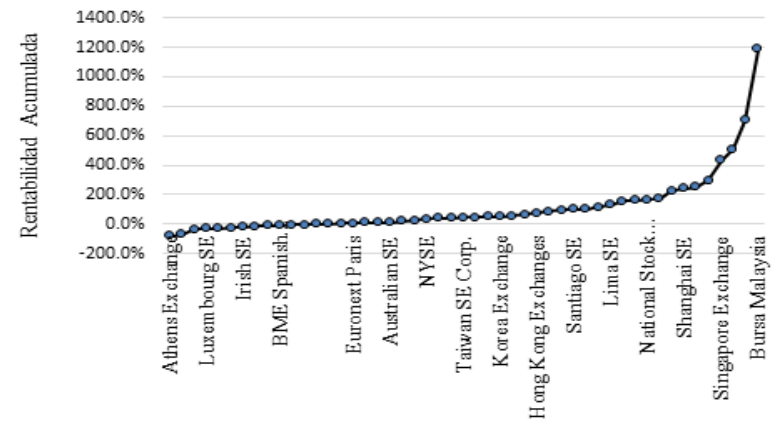

Figura1. Rentabilidad Acumulada por cada Bolsa de valores.

Los resultados obtenidos por las bolsas de valores más famosas como la Bolsa de Valores de Nueva York denominada el NYSE y su índice composite logro 33.3\%, mientras que el NASDAQ OMX con su índice composite obtuvo $116.3 \%$.

De acuerdo al concepto de rentabilidad promedio, que para una mejor comprensión los datos mensuales se han anualizado utilizando la capitalización compuesta, se ha obtenido que el índice más rentable ha sido la Bolsa de Viena Austria con su índice SE Price Index que obtuvo una rentabilidad promedio anual de $93.8 \%$, en segundo lugar, muy distantes de la primera, se ubica la bolsa de Suiza con índice SMI que obtuvo 34\% y en tercer lugar la bosa de Oslo con su índice OSEBXPR que obtuvo 32\%.
Las bolsas que obtuvieron menor rentabilidad fueron la Bolsa de Bermuda con su Índice BSX Index el cual resulto en una pérdida de $10.5 \%$, la Bolsa BM\&FBOVESPA Brasil con su índice Ibovespa con $-10 \%$ y la bolsa de Valores de Lima con su Índice IGBV con $-2 \%$.

Las Bolsas que obtuvieron rendimientos intermedios fueron la Bolsa Española de Madrid España con su índice IGBM, la Bolsa de Filipinas con su índice PSEi, la bolsa Española de Barcelona con su índice BCN Global-100 y la Bolsa de Atenas con su índice general todos con un 6\% de rentabilidad anual. En la Figura 2 y Cuadro 2 se puede observar las rentabilidades de todos los índices.

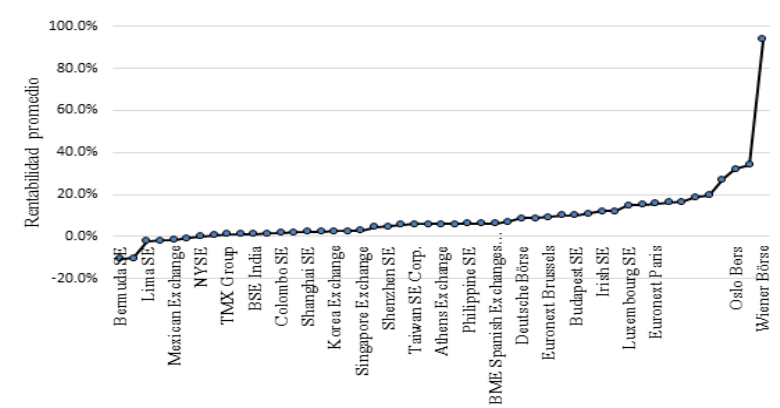

Fiugura 2. Rentabilidad promedio anualizada de las Bolsas de Valores en el Periodo 2006 -Junio 2015.

Cuadro 2. Rentabilidad promedio de Bolsas de Valores en el periodo 2006 - Junio 2015.

\begin{tabular}{|c|c|c|c|}
\hline Bolsa de Valores & Índice & Rp. mes & $\begin{array}{c}\mathrm{Rp} \\
\text { anual }\end{array}$ \\
\hline Bermuda SE & BSX Index & $-0.9 \%$ & $-10.5 \%$ \\
\hline BM\&FBOVESPA & Ibovespa & $-0.9 \%$ & $-10.1 \%$ \\
\hline Lima SE & $\begin{array}{l}\text { Índice General } \\
\text { BVL (IGBVL) }\end{array}$ & $-0.2 \%$ & $-2.0 \%$ \\
\hline NASDAQ OMX & Composite & $-0.2 \%$ & $-2.0 \%$ \\
\hline Mexican Exchange & IPC CompMx & $-0.1 \%$ & $-1.7 \%$ \\
\hline Buenos Aires SE & Composite & $-0.1 \%$ & $-0.6 \%$ \\
\hline NYSE & Composite & $0.0 \%$ & $0.2 \%$ \\
\hline Santiago SE & IGPA & $0.1 \%$ & $0.7 \%$ \\
\hline TMX Group & $\begin{array}{l}\text { S\&P/TSX } \\
\text { Composite }\end{array}$ & $0.1 \%$ & $1.1 \%$ \\
\hline Australian SE & $\begin{array}{l}\text { All Ordinary } \\
\text { Price }\end{array}$ & $0.1 \%$ & $1.2 \%$ \\
\hline BSE India & S\&P BSE 500 & $0.1 \%$ & $1.3 \%$ \\
\hline Bursa Malaysia & $\begin{array}{l}\text { FBM Emas } \\
\text { Index }\end{array}$ & $0.1 \%$ & $1.4 \%$ \\
\hline Colombo SE & CSE All Share & $0.1 \%$ & $1.7 \%$ \\
\hline $\begin{array}{l}\text { Hong Kong } \\
\text { Exchanges }\end{array}$ & $\begin{array}{c}\text { S\&P/HKEX } \\
\text { LargeCap } \\
\text { Index } \\
\text { SSE }\end{array}$ & $0.2 \%$ & $2.1 \%$ \\
\hline Shanghai SE & $\begin{array}{l}\text { Composite } \\
\text { Index }\end{array}$ & $0.2 \%$ & $2.3 \%$ \\
\hline $\begin{array}{l}\text { Japan Exchange } \\
\text { Group - Tokyo }\end{array}$ & TOPIX & $0.2 \%$ & $2.4 \%$ \\
\hline Korea Exchange & KOSPI & $0.2 \%$ & $2.5 \%$ \\
\hline
\end{tabular}


Continuación del cuadro 2

\begin{tabular}{|c|c|c|c|}
\hline $\begin{array}{l}\text { National Stock } \\
\text { Exchange India }\end{array}$ & S\&P CNX 500 & $0.2 \%$ & $2.5 \%$ \\
\hline Singapore Exchange & $\begin{array}{l}\text { Straits Times } \\
\text { Index }\end{array}$ & $0.2 \%$ & $3.0 \%$ \\
\hline BME Spanish & Índice Bolsa & $0.4 \%$ & $4.5 \%$ \\
\hline Exchanges Bilbao & $\begin{array}{l}\text { Bilbao } 1999 \\
\text { SZSE }\end{array}$ & $0.4 \%$ & $4.5 \%$ \\
\hline Shenzhen SE & Composite & $0.4 \%$ & $4.7 \%$ \\
\hline Indonesia SE & $\begin{array}{l}\text { Index } \\
\text { JSX Composite } \\
\text { Index }\end{array}$ & $0.5 \%$ & $5.6 \%$ \\
\hline Taiwan SE Corp. & TAIEX & $0.5 \%$ & $5.8 \%$ \\
\hline $\begin{array}{l}\text { The Stock Exchange } \\
\text { of Thailand }\end{array}$ & SET Index & $0.5 \%$ & $6.0 \%$ \\
\hline Athens Exchange & General Price & $0.5 \%$ & $6.1 \%$ \\
\hline $\begin{array}{l}\text { BME Spanish } \\
\text { Exchanges } \\
\text { Barcelona }\end{array}$ & $\begin{array}{l}\text { BCN Global - } \\
100 \text { Index }\end{array}$ & $0.5 \%$ & $6.1 \%$ \\
\hline Philippine SE & $\begin{array}{l}\text { PSE Index } \\
\text { (PSEi) }\end{array}$ & $0.5 \%$ & $6.1 \%$ \\
\hline $\begin{array}{l}\text { BME Spanish } \\
\text { Exchanges Madrid }\end{array}$ & IGBM Index & $0.5 \%$ & $6.2 \%$ \\
\hline $\begin{array}{l}\text { BME Spanish } \\
\text { Exchanges Valencia }\end{array}$ & IGBV Index & $0.5 \%$ & $6.2 \%$ \\
\hline Borsa Istanbul & ISE 100 Index & $0.6 \%$ & $7.1 \%$ \\
\hline Deutsche Börse & CDAX Price & $0.7 \%$ & $8.7 \%$ \\
\hline Egyptian Exchange & EGX 30 Index & $0.7 \%$ & $8.8 \%$ \\
\hline Euronext Brussels & General Price & $0.8 \%$ & $9.4 \%$ \\
\hline $\begin{array}{l}\text { Euronext } \\
\text { Amsterdam }\end{array}$ & AAX & $0.8 \%$ & $10.1 \%$ \\
\hline Budapest SE & BUMIX & $0.8 \%$ & $10.2 \%$ \\
\hline Borsa Italiana & $\begin{array}{l}\text { FTSE Italia } \\
\text { MIB Storico }\end{array}$ & $0.9 \%$ & $10.9 \%$ \\
\hline Irish SE & ISEQ Overall & $1.0 \%$ & $12.1 \%$ \\
\hline Johannesburg SE & $\begin{array}{l}\text { FTSE/JSE All } \\
\text { Share }\end{array}$ & $1.0 \%$ & $12.1 \%$ \\
\hline Luxembourg SE & $\begin{array}{l}\text { Lux General } \\
\text { Price }\end{array}$ & $1.2 \%$ & $14.9 \%$ \\
\hline Euronext Lisbon & BVL General & $1.2 \%$ & $15.2 \%$ \\
\hline Euronext Paris & SBF 250 & $1.2 \%$ & $15.6 \%$ \\
\hline Malta SE & $\begin{array}{l}\text { MSE Share } \\
\text { Index }\end{array}$ & $1.3 \%$ & $16.4 \%$ \\
\hline $\begin{array}{l}\text { NASDAQ OMX } \\
\text { Nordic Stockholm }\end{array}$ & OMXS & $1.3 \%$ & $16.5 \%$ \\
\hline $\begin{array}{l}\text { NASDAQ OMX } \\
\text { Nordic Copenhagen }\end{array}$ & OMXC & $1.4 \%$ & $18.8 \%$ \\
\hline $\begin{array}{l}\text { NASDAQ OMX } \\
\text { Nordic Helsinki }\end{array}$ & OMXH & $1.5 \%$ & $19.5 \%$ \\
\hline Tel Aviv SE & General & $2.0 \%$ & $27.2 \%$ \\
\hline Oslo Bers & OSEBXPR & $2.4 \%$ & $32.3 \%$ \\
\hline $\begin{array}{l}\text { SIX Swiss } \\
\text { Exchange }\end{array}$ & SMI & $2.5 \%$ & $34.0 \%$ \\
\hline Wiener Börse & SE Price Index & $5.7 \%$ & $93.8 \%$ \\
\hline
\end{tabular}

Elaboración propia

Las diferencias entre rentabilidad promedio y rentabilidad acumulada se deben a que la rentabilidad promedio, al ser un promedio, considera las subidas y caídas de precio en cada periodo, es decir las rentabilidades positivas y negativas. Por otro lado, la rentabilidad acumulada solo considera el cambio de precios entre el inicio y el final del periodo considerado. Esta es la razón por la cual se observan diferencias en el ranking al utilizar el concepto de rentabilidad promedio y rentabilidad acumulada.

\subsection{Riesgo}

En cuanto al riesgo que presentan los índices de las Bolsas de Valores se obtuvieron los siguientes resultados.

Los índices que presentan la mayor variabilidad medido según la desviación estándar de su rentabilidad fueron el SE Price de la Bolsa de Viena con un valor de 0.53 de riesgo, tomar en cuenta que este índice fue también el que presento la mayor rentabilidad, después sigue del índice SMI de la bolsa de Suiza con un valor de 0.2 y en tercer lugar lo ocupa el índice Ibovespa de la bolsa BM\&FBOVESPA de Sao Paulo Brasil con una desviación estándar de 0.10 .

Los índices que presentan los menores riesgos son el índice Straits Times de la Bolsa de Valores de Singapur con 0.037 de desviación estándar, el índice IGVB de la Bolsa de Lima Perú con 0.039 y el índice CDAX Price de la Bolsa de la Bolsa de Frankfurt Alemania con 0.04

En el Cuadro 3 y Figura 3 se pueden observar las desviaciones estándar de todos los índices.

Cuadro 3. Riesgo de las Bolsas en el periodo 2006 Junio 2015.

\begin{tabular}{|c|c|c|}
\hline Bolsa de Valores & Índice & $\begin{array}{l}\text { Desv. } \\
\text { Stand. }\end{array}$ \\
\hline Singapore Exchange & Straits Times Index & 0.03784 \\
\hline Lima SE & $\begin{array}{l}\text { Índice General } \\
\text { (IGBVL) }\end{array}$ & 0.03977 \\
\hline Deutsche Börse & CDAX Price & 0.04041 \\
\hline Shanghai SE & SSE Composite Index & 0.04149 \\
\hline Johannesburg SE & FTSE/JSE All Share & 0.04432 \\
\hline $\begin{array}{l}\text { BME Spanish } \\
\text { Exchanges Madrid }\end{array}$ & IGBM Index & 0.04654 \\
\hline Indonesia SE & JSX Composite Index & 0.04747 \\
\hline $\begin{array}{l}\text { BME Spanish } \\
\text { Exchanges Bilbao }\end{array}$ & $\begin{array}{l}\text { Índice Bolsa Bilbao } \\
1999\end{array}$ & 0.04756 \\
\hline $\begin{array}{ll}\text { National } & \text { Stock } \\
\text { Exchange India } & \end{array}$ & S\&P CNX 500 & 0.04775 \\
\hline Irish SE & ISEQ Overall & 0.04814 \\
\hline Hong Kong Exchanges & $\begin{array}{l}\text { S\&P/HKEX } \\
\text { LargeCapI. }\end{array}$ & 0.04878 \\
\hline Borsa Istanbul & ISE 100 Index & 0.05045 \\
\hline Budapest SE & BUMIX & 0.05059 \\
\hline Bermuda SE & BSX Index & 0.0516 \\
\hline Euronext Brussels & General Price & 0.05185 \\
\hline Korea Exchange & KOSPI & 0.05242 \\
\hline Taiwan SE Corp. & TAIEX & 0.05359 \\
\hline BSE India & S\&P BSE 500 & 0.05362 \\
\hline
\end{tabular}


Continuación del cuadro 3

\begin{tabular}{|c|c|c|}
\hline $\begin{array}{l}\text { BME Spanish } \\
\text { Exchanges Valencia }\end{array}$ & IGBV Index & 0.0546 \\
\hline Bursa Malaysia & FBM Emas Index & 0.05469 \\
\hline $\begin{array}{l}\text { NASDAQ OMX Nordic } \\
\text { Stockholm }\end{array}$ & OMXS & 0.05596 \\
\hline Colombo SE & CSE All Share & 0.05648 \\
\hline Santiago SE & IGPA & 0.05678 \\
\hline $\begin{array}{l}\text { The Stock Exchange of } \\
\text { Thailand }\end{array}$ & SET Index & 0.05802 \\
\hline Mexican Exchange & IPC CompMx & 0.05815 \\
\hline Athens Exchange & General Price & 0.05998 \\
\hline Australian SE & All Ordinary Price & 0.06029 \\
\hline Euronext Amsterdam & AAX & 0.06163 \\
\hline NYSE & Composite & 0.06202 \\
\hline Shenzhen SE & $\begin{array}{l}\text { SZSE Composite } \\
\text { Index }\end{array}$ & 0.0621 \\
\hline $\begin{array}{l}\text { Japan Exchange Group } \\
\text { - Tokyo }\end{array}$ & TOPIX & 0.06324 \\
\hline $\begin{array}{l}\text { BME Spanish } \\
\text { Exchanges Barcelona }\end{array}$ & $\begin{array}{l}\text { BCN Global - } 100 \\
\text { Index }\end{array}$ & 0.06329 \\
\hline NASDAQ OMX & Composite & 0.06335 \\
\hline Egyptian Exchange & EGX 30 Index & 0.06387 \\
\hline $\begin{array}{l}\text { NASDAQ OMX Nordic } \\
\text { Copenhag. }\end{array}$ & OMXC & 0.06438 \\
\hline TMX Group & S\&P/TSX Composite & 0.06626 \\
\hline Malta SE & MSE Share Index & 0.0668 \\
\hline Buenos Aires SE & Composite & 0.07385 \\
\hline Luxembourg SE & Lux General Price & 0.07615 \\
\hline Euronext Lisbon & BVL General & 0.07648 \\
\hline Borsa Italiana & $\begin{array}{l}\text { FTSE Italia MIB } \\
\text { Storico }\end{array}$ & 0.07966 \\
\hline $\begin{array}{l}\text { NASDAQ OMX Nordic } \\
\text { Helsinki }\end{array}$ & OMXH & 0.08992 \\
\hline Tel Aviv SE & General & 0.09066 \\
\hline Philippine SE & PSE Index (PSEi) & 0.09338 \\
\hline Euronext Paris & SBF 250 & 0.09691 \\
\hline Oslo Bers & OSEBXPR & 0.09955 \\
\hline BM\&FBOVESPA & Ibovespa & 0.10154 \\
\hline SIX Swiss Exchange & SMI & 0.2009 \\
\hline Wiener Börse & SE Price Index & 0.53059 \\
\hline
\end{tabular}

Elaboración propia

El índice AAX de la Bolsa de Valores Euronext de Amsterdam con una desviación estándar de 0.062, el índice All ordinary Price de la Bolsa de Australia con 0.060, el índice General Price de la Bolsa de Atenas con 0.059 y el índice IPC CompMx de la Bolsa Mexicana con 0.058 fueron los que presentaron riesgo intermedio.
Continuación del cuadro 3

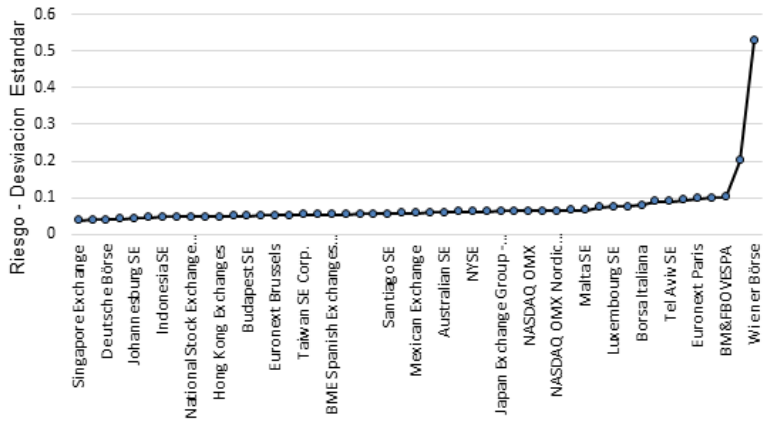

Figura 3. Riesgo en las Bolsas de Valores en el periodo 2006 -Junio 2015, segun la desviacion estandar.

Al medir el riesgo como coeficiente de variabilidad se obtuvo que los índices más riesgos son el OSEBXPR de la Bolsa de Oslo que presenta un coeficiente de variabilidad de 0.237 y los índices OMXS y OMXC de las Bolsas Nasdaq de Estocolmo y la de Copenhague con 0.229 y 0.225 de coeficiente de variabilidad respectivamente.

Los índices menos riesgosos, de acuerdo a su coeficiente de variabilidad, fueron: el índice BSX de la Bolsa de Bermuda con -0.17, el índice Ibovespa de la Bolsa BM\&FBOVESPA con -0.086 y el índice IGBV de la Bolsa de Lima Perú con -0.42 de coeficiente de variabilidad.

Los índice de riesgo intermedio, de acuerdo a su coeficiente de variabilidad, fueron el índice de JSX Composite de la Bolsa de indonesia, el índice IGBV del Bolsa BME de Madrid, el índice TAIEX de la Bolsa de Taiwán y el índice SET de la Bolsa de Tailandia con un coeficiente de variabilidad de 0.0957, 0.092, 0.0885 y .0837 respectivamente.

Los coeficientes de variabilidad para todas la índices de Bolsa se pueden observar en el Cuadro 4 y figura 4.

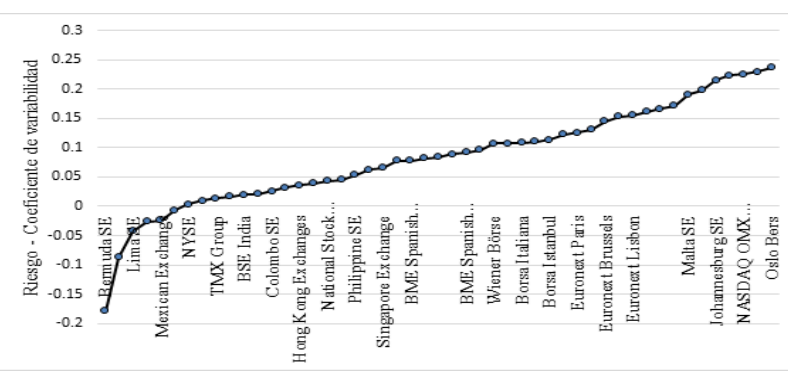

Figura 4. Riesgo de las Bolsas de valores en el periodo 2006 - Junio 2015, segun el coeficiente de variabilidad.

Cuadro 4. Riesgo de las Bolsas de Valores en el periodo 2006 - Junio 2015.

\begin{tabular}{lcc}
\hline Bolsa de Valores & Índice & Coef. Var. \\
\hline Bermuda SE & BSX Index & -0.17871 \\
BM\&FBOVESPA & Ibovespa & -0.08683 \\
\hline
\end{tabular}




\begin{tabular}{|c|c|c|}
\hline \multicolumn{3}{|l|}{ Continuación del cuadro 4} \\
\hline Lima SE & $\begin{array}{l}\text { Índice General } \\
\text { BVL (IGBVL) }\end{array}$ & -0.04289 \\
\hline NASDAQ OMX & Composite & -0.02601 \\
\hline Mexican Exchange & IPC CompMx & -0.02406 \\
\hline Buenos Aires SE & Composite & -0.00692 \\
\hline NYSE & Composite & 0.002919 \\
\hline Santiago SE & IGPA & 0.009865 \\
\hline TMX Group & $\begin{array}{l}\text { S\&P/TSX } \\
\text { Composite }\end{array}$ & 0.013671 \\
\hline Australian SE & All Ordinary Price & 0.016815 \\
\hline BSE India & S\&P BSE 500 & 0.0194 \\
\hline Bursa Malaysia & FBM Emas Index & 0.020959 \\
\hline Colombo SE & CSE All Share & 0.025404 \\
\hline $\begin{array}{l}\text { Japan Exchange Group - } \\
\text { Tokyo }\end{array}$ & TOPIX & 0.031823 \\
\hline Hong Kong Exchanges & $\begin{array}{c}\text { S\&P/HKEX } \\
\text { LargeCap Index }\end{array}$ & 0.035962 \\
\hline Korea Exchange & KOSPI & 0.039341 \\
\hline $\begin{array}{l}\text { National Stock Exchange } \\
\text { India }\end{array}$ & S\&P CNX 500 & 0.043321 \\
\hline Shanghai SE & $\begin{array}{l}\text { SSE Composite } \\
\text { Index }\end{array}$ & 0.04483 \\
\hline Philippine SE & PSE Index (PSEi) & 0.05307 \\
\hline Shenzhen SE & $\begin{array}{l}\text { SZSE Composite } \\
\text { Index }\end{array}$ & 0.062272 \\
\hline Singapore Exchange & Straits Times Index & 0.065745 \\
\hline $\begin{array}{l}\text { BME Spanish Exchanges } \\
\text { Barcelona }\end{array}$ & $\begin{array}{l}\text { BCN Global - } 100 \\
\text { Index }\end{array}$ & 0.077883 \\
\hline $\begin{array}{l}\text { BME Spanish ExchangesÍ } \\
\text { Bilbao }\end{array}$ & $\begin{array}{l}\text { Índice Bolsa Bilbao } \\
1999\end{array}$ & 0.077974 \\
\hline Athens Exchange & General Price & 0.081855 \\
\hline $\begin{array}{l}\text { The Stock Exchange of } \\
\text { Thailand }\end{array}$ & SET Index & 0.083749 \\
\hline Taiwan SE Corp. & TAIEX & 0.088526 \\
\hline $\begin{array}{l}\text { BME Spanish Exchanges } \\
\text { Valencia }\end{array}$ & IGBV Index & 0.092001 \\
\hline Indonesia SE & $\begin{array}{l}\text { JSX Composite } \\
\text { Index }\end{array}$ & 0.095697 \\
\hline Wiener Börse & SE Price Index & 0.106833 \\
\hline $\begin{array}{l}\text { BME Spanish Exchanges } \\
\text { Madrid }\end{array}$ & IGBM Index & 0.107641 \\
\hline Borsa Italiana & $\begin{array}{l}\text { FTSE Italia MIB } \\
\text { Storico }\end{array}$ & 0.108484 \\
\hline Egyptian Exchange & EGX 30 Index & 0.110445 \\
\hline Borsa Istanbul & ISE 100 Index & 0.112975 \\
\hline SIX Swiss Exchange & SMI & 0.122964 \\
\hline Euronext Paris & SBF 250 & 0.125571 \\
\hline Euronext Amsterdam & AAX & 0.130487 \\
\hline Euronext Brussels & General Price & 0.145265 \\
\hline Luxembourg SE & Lux General Price & 0.152847 \\
\hline Euronext Lisbon & BVL General & 0.154923 \\
\hline Budapest SE & BUMIX & 0.161104 \\
\hline $\begin{array}{l}\text { NASDAQ OMX Nordic } \\
\text { Helsinki }\end{array}$ & OMXH & 0.166601 \\
\hline Deutsche Börse & CDAX Price & 0.171665 \\
\hline Malta SE & MSE Share Index & 0.190468 \\
\hline Irish SE & ISEQ Overall & 0.198057 \\
\hline
\end{tabular}

Continuación del cuadro 4

\begin{tabular}{lcc}
\hline Johannesburg SE & $\begin{array}{c}\text { FTSE/JSE All } \\
\text { Share }\end{array}$ & 0.215672 \\
Tel Aviv SE & General & 0.223213 \\
NASDAQ OMX Nordic & OMXC & 0.225016 \\
$\begin{array}{l}\text { Copenhagen } \\
\text { NASDAQ OMX Nordic }\end{array}$ & OMXS & 0.229136 \\
$\begin{array}{l}\text { Stockholm } \\
\text { Oslo Bers }\end{array}$ & OSEBXPR & 0.236836 \\
\hline
\end{tabular}

Elaboración propia

\subsection{Comparacion de rentabilidad y riesgo por region}

Tal como se puede apreciar en el Cuadro 5, la región Asia Pacifico es la presenta los mayores niveles de rentabilidad promedio anualizada de $18.28 \%$, mientras que la región Américas tiene $8.15 \%$ y la de menor rentabilidad la región es la región Europa y África Medio Este con tan solo $2.85 \%$ de rentabilidad. Esta cifras son coincidentes con los datos de rentabilidad acumulada que fueron 264\% para la región Asia Pacifico posicionándolo en el primer lugar , 115\% para la región Américas que ocupo el segundo lugar y en tercer lugar 16\% para las Bolsas de la región Europa y África Medio Este. En el Cuadro No 1 se puede observar a que región corresponde cada una de las Bolsas.

Con respecto al riesgo, según la desviación estándar, la región Asia Pacifico presenta el mayor nivel de riesgos con 0.056 , la de riesgo intermedio es Europa y África Medio Este con 0.47 y mientras que Américas presenta el riesgo más bajo con 0.043 .

Al evaluar el riesgo, según el coeficiente de variabilidad, se obtuvo que la región Asia Pacifico presenta el mayor nivel de riesgos más alto con 0.25 , Américas presenta un riesgo intermedio de 0.15 y la de menor riesgo es la región Europa y África Medio Este con 0.049.

Al contrastar el riesgo y rentabilidad entre las regiones se puede afirmar que a nivel de regiones se presenta una relación positiva entre riesgo y rentabilidad.

\subsection{Asociación entre riesgo y rentabilidad.}

Para este análisis se ha considerado a la rentabilidad promedio como medida de la rentabilidad y también a la desviación estándar como medidor de riesgo. A continuación, en la Figura 5, se muestra presenta la dispersión de datos y la recta de regresión línea obtenida. 


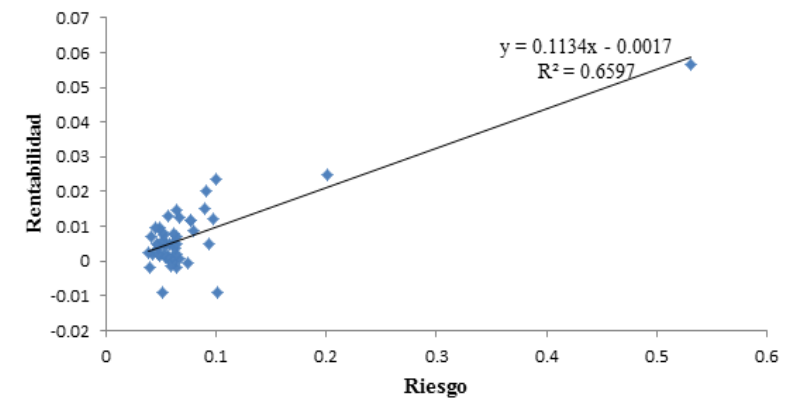

Figura 5. Diagrama de dispersion de rentabilidad y riesgo y ecuacion de regresion lineal.

El modelo de regresión lineal, asociación lineal, entre los dos variables rentabilidad y riesgo obtenido fue:

Rentabilidad $=\mathbf{- 0 . 0 0 1 7}+\mathbf{0 . 1 1 3 4} *$ Riesgo

Dónde:

- El intercepto $\beta 0$ estimado es - 0.0017

- La pendiente $\beta 1$ estimado es 0.1134

Para evaluar la bondad de este modelo de regresión lineal se ha determinado que existe una correlación lineal positiva de $\mathrm{R}=0.8122$ entre la variable independiente riesgo y la variable dependiente rentabilidad, tal como se observa en el Anexo 1.

Cuadro 5. Rentabilidad de las Bolsas según regiones.

\begin{tabular}{lccccc}
\hline Region & $\begin{array}{c}\text { R pro. } \\
\text { mes }\end{array}$ & $\begin{array}{c}\text { R pro. } \\
\text { anual }\end{array}$ & $\begin{array}{c}\text { Rentabilidad } \\
\text { Acumulada. }\end{array}$ & Estandar & Variabilidad \\
\hline EAME & $0.23 \%$ & $2.85 \%$ & $16 \%$ & 0.04729 & 0.04951 \\
Americas & $0.65 \%$ & $8.15 \%$ & $115 \%$ & 0.04321 & 0.15153 \\
Asia & $1.41 \%$ & $18.28 \%$ & $264 \%$ & 0.05622 & 0.25061 \\
Pacífico & & & & &
\end{tabular}

para afirmar que el valor del intercepto es -0.0017.

La pendiente de la ecuación de regresión lineal calculada $\beta 1=0.1134$ significa que por cada $10 \%$ de riesgo adicional que se asume por invertir en los índices de las Bolsas de Valores se puede lograr una rentabilidad adicional mensual de 1.134\%. En valores anualizados esto implica una rentabilidad adicional mensual de $14.48 \%$ por cada $10 \%$ de riesgo adicional.

Para probar la validez de la pendiente estimada, se ha utilizado una prueba t. Para esta prueba considerando un nivel de significancia de 5\%, se obtuvo un valor $\mathrm{t}$ de tabla de 2.0117. El valor calculado de t es 9.5463, ver Anexo 1, como este valore es mayor que el valor $\mathrm{t}$ de tabla, podemos afirmar que no existe suficiente evidencia para afirmar que la pendiente sea igual a cero; es decir, la pendiente es igual a 0.1134 y que evidencia permite afirmar que el riesgo afecta la rentabilidad. Los mismos resultados se obtienen con el valor-p que resulto 1.4E12, es decir un número mucho menor que el nivel de significancia de 0.05 . También, lo mismo ocurre con el valor $\mathrm{F}$ donde el $\mathrm{F}$ calculado es 91.13 y el $\mathrm{F}$ según tabla es del estadístico con 1 y 16 grados de libertad es 4.0493 mayores detalles se pueden ver el Anova del Anexo 1.

El estadístico Durbin Watson fue 0.34; sin embargo, Para el caso de variables como rentabilidad donde el cálculo se basa en la variación del año actual con respecto al anterior es posible tener la presencia de auto correlación.

Para realizar la prueba acerca de los supuestos del modelo de regresión obtenido, se ha analizado los residuales que se muestran en la Figura 6. En esta figura se puede observar que los datos no presentan una tendencia definida, por lo tanto podemos afirmar que el modelo obtenido no viola las principales asunciones como linealidad, independencia de errores, normalidad del error y homocedasticidad.

Elaboración propia

Asimismo se ha determinado el coeficiente de determinación, el cual para el modelo planteado representó un valor de R2 igual 0.6597 tal como se puede observar en el Anexo1. Esto quiere decir que el 65.97\% de variabilidad en la variable dependiente rentabilidad es explicado por la variable independiente riesgo. Por lo tanto podemos afirmar que existe una relación lineal positiva entre rentabilidad y riesgo. Esto quiere decir que para obtener un mayor nivel de rentabilidad debemos asumir un nivel mayor de riesgo.

El intercepto estimado $\beta 0=-0.0017$ significa que la rentabilidad mínima para invertir en los índices de las bolsas de valores es prácticamente cero. Para probar la validez del estimado de intercepto se ha considerado el valor-p, el cual es igual a 0.1717 , el cual se puede observar en el Anexo 1. Este valor es mayor que 0.05 correspondiente al el valor de nivel de significancia. Por lo tanto podemos concluir que la evidencia no es suficiente

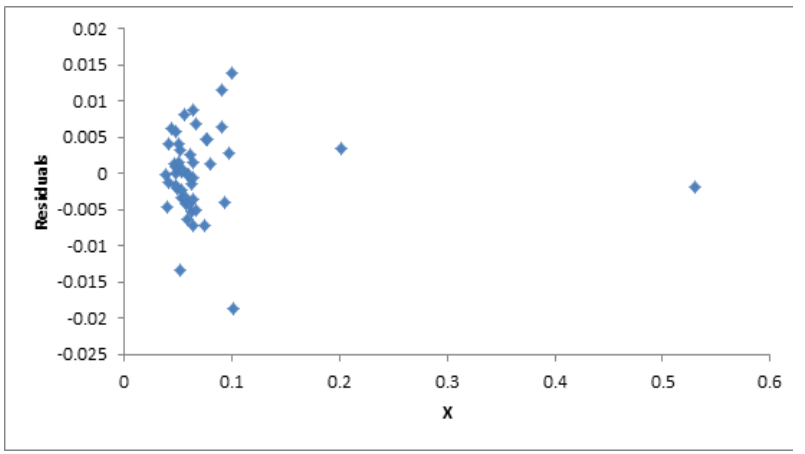

Figura 6. Diagrama de dispersion de residuales (Residual Plot). 


\section{Conclusiones}

Las Bolsas de valores que presentaron mayor rentabilidad promedio fueron la Bolsa de Viena, Suiza y Oslo. Las Bolsas de rentabilidad más baja fueron la Bolsa de Valores de Lima Perú, La BM\&FBOVESPA de Brasil y la de Bermudas. Los Bolsas que presentaron los mayor riesgo fueron las Bolsas de Viena, La Bolsa Suiza SIX, la Bolsa BM\&FBOVESPA de Brasil y la Bolsa de Oslo. Las Bolsas menos riesgosas fueron la bolsa Deutsche Börse de Alemania, las de Singapur y la de Lima Perú. Las Bolsas de valores de la región Asia Pacifico fueron las que presentaron los mayores niveles de rentabilidad, las Bolsas de Américas presentaron rentabilidad y riesgo medio y la de la región Europa y África Medio presentaron los niveles de riesgo y rentabilidad más baja. Finalmente, se puede concluir que existe una relación lineal positiva entre el riesgo y la rentabilidad de las Bolsas de valores.

\section{Literatura citada}

Brealey \& Myers, S. 2011. Fundamentos de Finanzas Corporativas, Mc Graw Hill. México.

Levine, Krehbiel, Berenson. 2103. Business statistics: A first course. Pearson.

Martin, M. A.. 2007. Mercado de Capitales. Thomson.

Ross, Westerfield y Jaffe. 2012. Finanzas Corporativas. McGraw Hill. México.

World Federation of Exchanges. 2013. Statistics Definition and Examples.

Referencias de internet:

PHstat12. 2013. Users Guide.

http://wps.aw.com/phstat/

World Federation of Exchange.

http://www.world-exchanges.org/

\section{Anexos}

Anexo 1. Resultados del cálculo de la bondad del modelo de regresión lineal.

\begin{tabular}{lc}
\hline Estadísticos de la regresión \\
R múltiple & 0.812249 \\
R cuadrado & \\
0.659748 & \\
R ajustado & 0.652509 \\
Error estándar & 0.005872 \\
Observaciones & 49
\end{tabular}

\begin{tabular}{|c|c|c|c|c|c|}
\hline \multicolumn{6}{|l|}{ ANOVA } \\
\hline & Gl. & SS & MS & $\mathrm{F}$ & Valor-p \\
\hline Regresión & 1 & 0.003143 & 0.003143 & 91.13304 & $1.4 \mathrm{E}-12$ \\
\hline Residual & 47 & 0.001621 & $3.45 \mathrm{E}-05$ & & \\
\hline Total & 48 & 0.004764 & & & \\
\hline
\end{tabular}

\begin{tabular}{|c|c|c|c|c|c|c|c|c|}
\hline & Coeficiente. & $\begin{array}{c}\text { Error } \\
\text { Estándar }\end{array}$ & t Calculado & & $\begin{array}{c}\text { Inferior } \\
95 \%\end{array}$ & $\begin{array}{c}\text { Superior } \\
95 \%\end{array}$ & $\begin{array}{c}\text { Inferior } \\
95 \%\end{array}$ & $\begin{array}{c}\text { Superior } \\
95 \%\end{array}$ \\
\hline$\beta 0$ Intercepto & -0.00166 & 0.001214 & -1.36845 & 0.177678 & -0.0041 & 0.000781 & -0.0041 & 0.000781 \\
\hline$\beta 1$ Pendiente & 0.113443 & 0.011883 & 9.546363 & $1.4 \mathrm{E}-12$ & 0.089536 & 0.137349 & 0.089536 & 0.137349 \\
\hline
\end{tabular}

Nivel Confiab. $\quad 0.95$

Valor Critico t $\quad 2.011741$

$\beta 0$ Ampl. Med. $\quad 0.002441$

$\beta 1$ Ampl. Med 0.023906

Fuente: Resultados de regresión de PHstat2 